\title{
Mobility of Data in Distributed Hybrid Computing Systems
}

\author{
Philippe Faes, Mark Christiaens, and Dirk Stroobandt \\ Ghent University, \\ SintPietersnieuwstraat 41, B9000 Ghent, Belgium
}

\begin{abstract}
In distributed hybrid computing systems, traditional sequential processors are loosely coupled with reconfigurable hardware for optimal performance. This loose coupling proves to be a communication challenge; the processor units cannot efficiently share a physical memory. This paper proposes a distributed shared memory architecture and a method for effective data migration within that shared memory. Data is moved using a novel garbage collection scheme, the dual semispace collector. The new garbage collector and the distributed memory prove to be an effective means of data migration in distributed hybrid computing systems.
\end{abstract}

\section{Introduction}

The proliferation of Field Programmable Gate Arrays (FPGAs) as reconfigurable processor units has enabled system architects to combine the computational power of loosely coupled Instruction Set Processors (ISPs) and FPGAs for applications with high computational demands. We call these systems distributed hybrid computing systems. Because of their loose coupling, the processor units cannot share one physical memory lest the communication overhead becomes a major bottleneck.

Instead of copying data explicitly between the processor units, we offer the illusion of a shared heap. In reality this heap is distributed over memory chips closely connected to the ISP and FPGA respectively. This paper presents a novel garbage collector algorithm, dual semispace, which can move objects within the heap from one physical memory to another. This operation is completely transparent for the programmer.

Unlike pagebased distributed memory systems, the garbage collector moves data per object. Objects form a

\footnotetext{
* Mark Christiaens is currently with Symantec Corporation, Antwerpsesteenweg 19, B9080 Lochristi, Belgium

$1424409101 / 07 / \$ 20.00 \quad$ (C) 2007 IEEE.
}

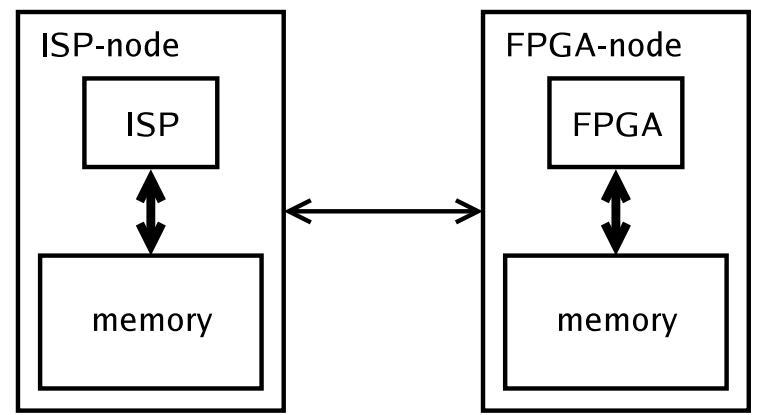

Figure 1. Physical interconnection of the ISPnode and the FPGA-node

semantic group of data, unlike memory pages, which form fixedsize groups of data. Our results show that the dual semispace garbage collector is an effective alternative for moving data in distributed hybrid computing systems. The dual semispace garbage collector is the first garbage collector, to the best of our knowledge, to move data transparently from one physical memory to another. It relieves the application programmers from dealing with data placement, encapsulating this responsibility in the runtime environment.

The rest of this paper is organized as follows. The next section introduces the problem of data mobility in hybrid distributed computing systems. Section 3 explains how the garbage collector is used for moving data inside a distributed computing system, and introduces the dual semispace garbage collector. Section 4 outlines a demonstration setup of our fully functional system, and presents timing results for a reallife application. Related work is outlined in Section 5. The paper concludes and looks forward to future work in Section 6.

\section{Data placement in distributed hybrid computing}

Our hybrid distributed computing system, as shown in Fig. 1, has two cooperating processor nodes: an ISP node and an FPGA node, connected through a relatively slow 
The remainder of this paper is not included as this paper is copyrighted material. If you wish to obtain an electronic version of this paper, please send an email to bib@elis.UGent.be with a request for publication P107.032.pdf. 